\title{
Surgical Repair of an Unstable Third Metatarsophalangeal Joint Dislocation via Direct Plantar Plate Repair: A Simple and Effective Technique
}

\author{
Christopher W Kinter, $M D^{1,2^{*}}$, Kevin J Kinter, $M S^{2}$ and Christopher W Hodgkins, $M D^{2,3}$ \\ ${ }^{1}$ Department of Orthopaedics, Emory University School of Medicine, USA \\ ${ }^{2}$ Herbert Wertheim College of Medicine, Florida International University, USA \\ ${ }^{3}$ Miami Orthopedics and Sports Medicine Institute, Baptist Health South Florida, USA
}

*Corresponding author: Christopher W Kinter, MD, Department of Orthopaedics, Emory University School of Medicine, Decatur, Georgia, USA

\begin{abstract}
Traumatic dislocation of a lesser metatarsophalangeal (MTP) joint is a rare case for a foot and ankle surgeon to encounter. These dislocations can be difficult to reduce due to the surrounding connective tissues that may be interposed in the joint space. These include the plantar plate, deep transverse metatarsal ligament, flexor tendons, and lumbricals. When the clinician is unable to reduce the phalanx, surgery is required to prevent sequelae such as further swelling, ecchymosis, joint damage, and impingement of the neurovascular bundle. In this case report, we present the case of a 53-year-old female who suffered a traumatic dislocation after a low-energy trip and fall injury. The MTP joint was found to be unstable, and therefore, we managed the dislocation through open reduction and direct repair of the plantar plate via a plantar approach. We feel the plantar plate repair makes our case unique as well as superior because it allows better visualization and obviates the need for further fixation with a K-wire.
\end{abstract}

\section{Keywords}

Metatarsophalangeal joint, Dislocation, Plantar plate, Open reduction, Plantar approach

\section{Abbreviations}

MTP: Metatarsophalangeal; BMI: Body Mass Index; MRI: Magnetic Resonance Imaging; VAS: Visual Analog Scale

\section{Introduction}

Dislocation of the metatarsophalangeal (MTP) joint is an uncommon injury. It is usually due to high energy impact directed at the base of the joint [1]. This typically occurs when the toes are extended and the ankle is slightly plantar flexed $[1,2]$. This force causes a dorsal dislocation of the MTP joint, which is far more common than plantar dislocation $[1,3,4]$. This dislocation is best treated by closed reduction, which is typically stable once reduced. However, there can be interposed ligaments and tendons that make this difficult. An irreducible MTP joint is typically handled surgically from a dorsal approach which requires pinning to maintain the reduction $[3,5,6]$. With a dorsal approach, it can also be difficult to visualize and repair plantar plate damage [7]. These challenges have driven the need for other surgical approaches. Reduction via a plantar approach can be less technically demanding and allows for full visualization of the plantar plate. Here we present the case of a low energy third MTP joint dislocation that was reducible but unstable, and explain how our use of the plantar approach resulted in stable fixation without the need for pinning.

\section{Case Description}

A 53-year-old female presented to our clinic three weeks after a low energy trip and fall in which she injured her left forefoot. A history and physical exam revealed obesity to be the patient's only comorbidity. She had a body mass index (BMI) of 45 . She originally went to an urgent care clinic. Radiographs at that time were 
normal. She was provided with a walking boot. She walked on the foot for several days, however she developed increased pain on the dorsal and plantar aspect of the third MTP joint, prompting her to go to an orthopedic walk-in clinic. She previously had a neuroma in her right foot with similar symptoms, so her present symptoms were attributed to this. A steroid injection was administered, which did not help alleviate her symptoms. A Magnetic Resonance Imaging (MRI) study was ordered, revealing a dislocated third MTP joint and associated plantar plate tear. She went back to the urgent care clinic where multiple unsuccessful attempts were made to reduce the dislocated third MTP joint.

At 3 weeks post-injury, she presented to our office. She was full-weight-bearing in a walking boot with swelling, tenderness, and decreased motion of her third MTP joint. The toe appeared laterally displaced and elevated. Radiographs revealed a dorsal dislocation of the third MTP joint (Figure 1). The toe was reducible but unstable. We attempted to hold the

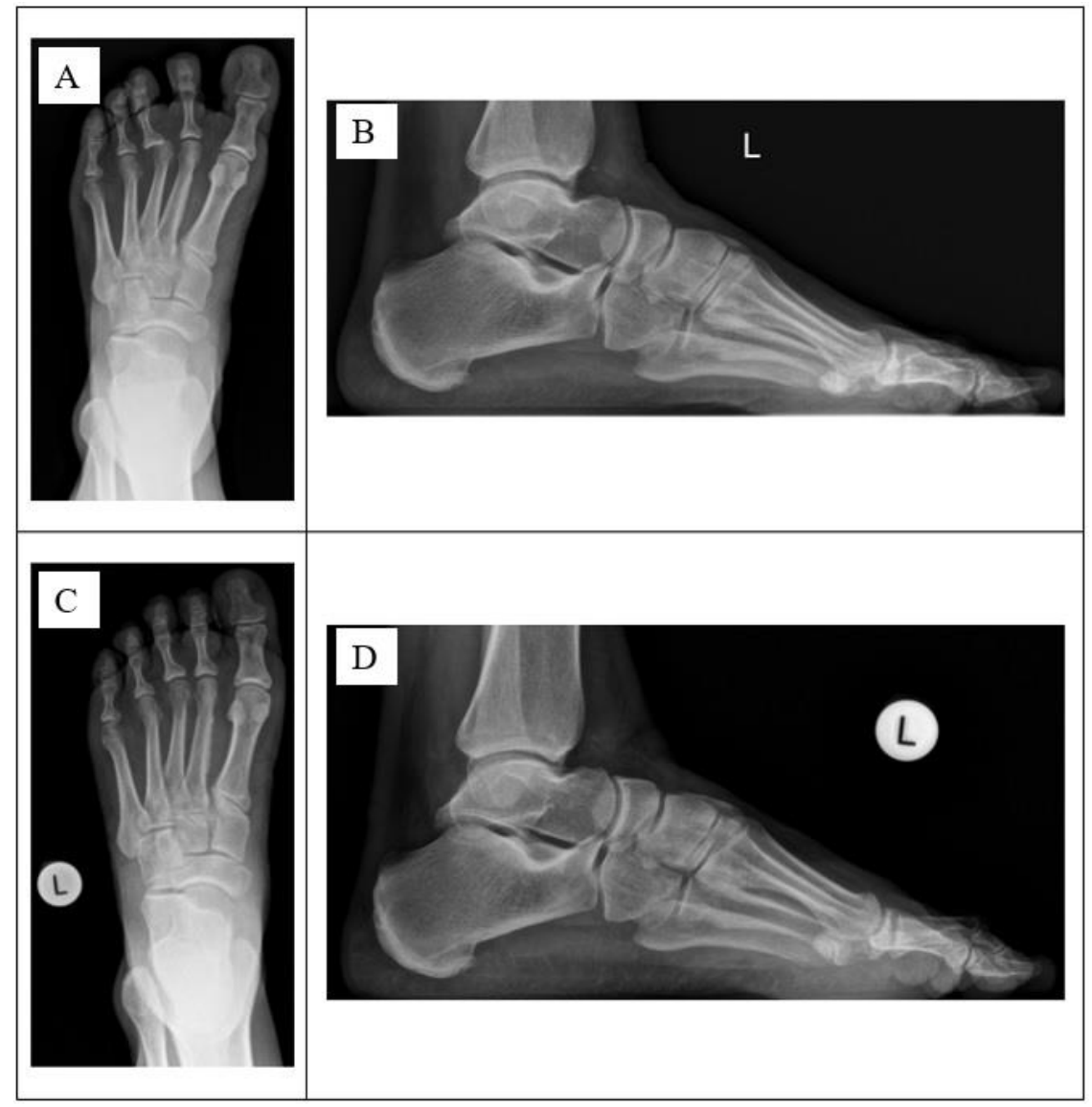

Figure 1: Radiographs of the left foot demonstrating dorsal dislocation of the $3^{\text {rd }}$ MTP joint. A) Preoperative AP; B) Preoperative Lateral; C) Postoperative AP; D) Postoperative lateral.
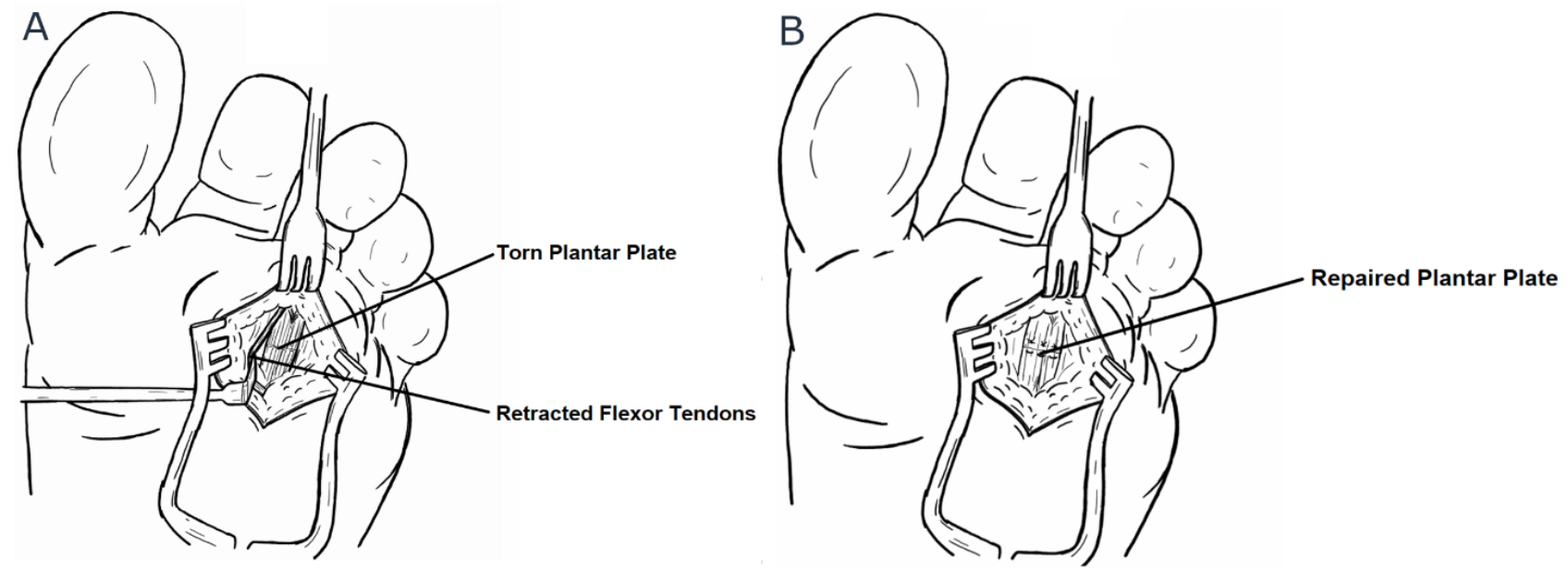

Figure 2: A) Illustration showing torn plantar plate; B) Illustration showing repaired plantar plate sutured in a horizontal mattress fashion with three interrupted positions. 
reduction with tape, however this was unsuccessful. We proceeded with open reduction and direct plantar plate repair using a plantar approach. During the procedure, careful dissection was carried out down to the flexor tendon sheath which was incised so both flexor tendons could be retracted. We discovered a horizontal tear in the plantar plate (Figure 2A). We repaired this with a fiber loop suture in a horizontal mattress fashion with 3 interrupted positions (Figure 2B). This held the toe reduced easily and did not require a k-wire to maintain the reduction, as can be noted in Figure 3. After closing, we sutured the third toe to the adjacent toes with it in a subtly plantar flexed position and applied a dorsiflexion-block dressing.

This syndactylization and dressing served to stabilize the toes in the early stages of healing. Following the procedure, she was instructed to be heel weight bearing in a post-operative shoe for 6 weeks. She returned to clinic at regular intervals to ensure wound healing and maintenance of reduction. After two weeks, the sutures to the adjacent toes and the plantar sutures were removed. Following this, we encouraged her to tape the toe in a plantar flexed position to reduce tension on the repair. At 6 weeks, her healing was unremarkable aside from some hyperesthesia at the plantar incision site, so we began gradually increasing weight-bearing through the forefoot.

At 3-month follow-up, the toe was still reduced, albeit with subtle radiographic lateral subluxation. She still had pain of 2 out of 10 on the Visual Analog Scale (VAS) and still had some hypersensitivity of the incision, which we attributed to digital nerve irritation.

In her most recent visit, at 1-year post-operation, she is now pain-free without any nerve pain. The slight lateral subluxation persists, as can be seen in Figure 4. She is using a custom metatarsal pad orthotic and avoiding barefoot walking or high impact activity until she can lose some weight.

\section{Discussion}

There is limited literature discussing how to handle a traumatically dislocated MTP joint. The most common MTP joint dislocation described is of the first MTP joint, with few reported cases of dislocated lesser MTP joints $[1,5,8]$. The mechanics of this injury are usually due to an impact to the base of the MTP joint causing hyperextension and dorsal dislocation $[1,3,5]$. High energy impact with the ground or impact of the foot during a motor vehicle accident are commonly described causes $[3,5]$. These dislocations typically occur in the dorsal direction due to weaker connective tissue, in the dorsal aspect of the MTP [1]. In addition, MTP

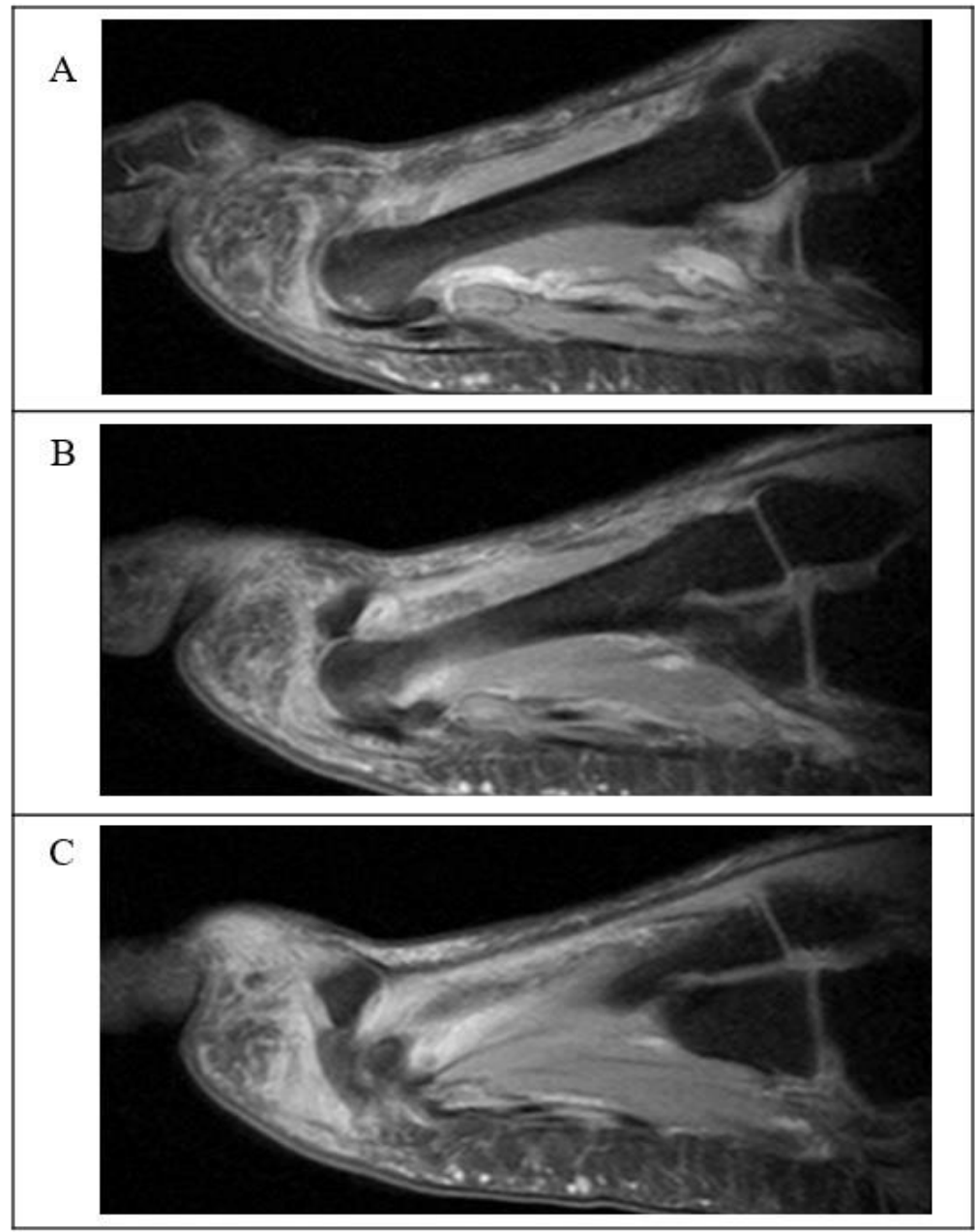

Figure 3: Preoperative MRI of the left foot showing the dorsal dislocation of the third MTP joint and a plantar plate tear. 

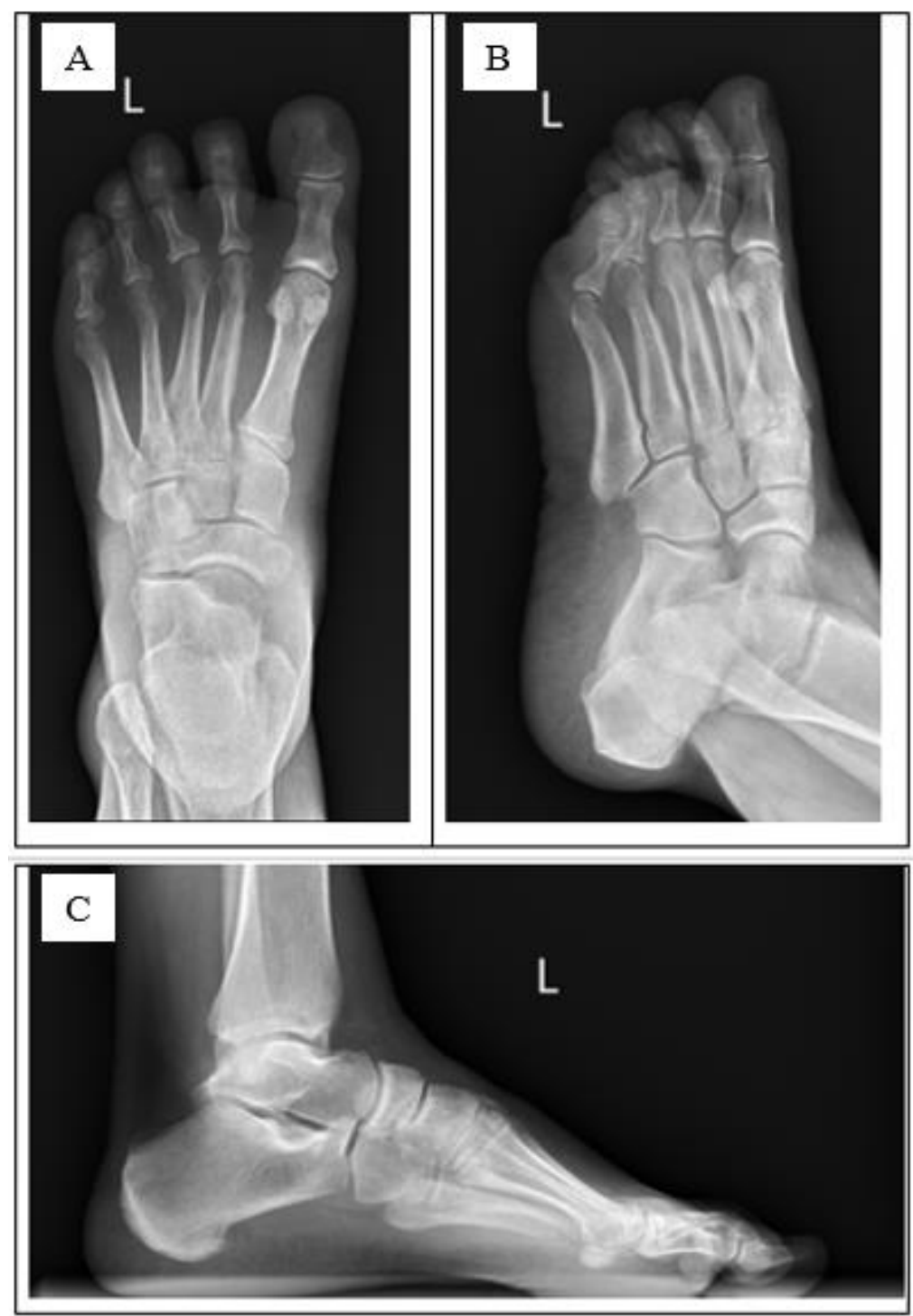

Figure 4: Radiographs at 1-year post-operation showing subluxation of the 3rd MTP joint. A) Anteroposterior; B) Oblique; C) Lateral.

joint dislocations are always associated with a plantar plate tear $[3,9]$. This tear allows for dorsal translation of the proximal phalanx upon the metatarsal head $[3,9]$. Hibino, et al. performed a cadaveric study where they determined that without damage to the plantar plate, dislocation of the MTP joint was not possible [3]. In our case, the injury appears to be due to a low energy trip and fall that caused an initial tear of the plantar plate. The tear in the plantar plate could have been further weakened due to a corticosteroid injection into the MTP joint, allowing the dislocation to occur [10].

Optimally, these injuries are addressed by manual reduction of the phalanx. A classic method described by Sir John Charnley for reducing a dislocated MTP joint is performed by hyper dorsiflexing the joint and applying axial traction to pull the proximal phalanx just distal to the metatarsal head [1]. Often with the lesser phalanges, it is difficult to maintain an adequate grip to perform this technique. One solution is to tie a bandage such as a Kerlix around the toe and pull axial traction while pushing posteriorly on the anterior aspect of the tibia
[11]. After successful reduction, the physician should evaluate the integrity of the ligaments to ensure stability and strength [8]. Further stability can be achieved through taping the toe in a neutral position and allowing capsular scar tissue to develop over several months if only minor damage is present [10]. However, often the phalanx is irreducible $[2-5,12,13]$. This is due to the possible interposition of the many surrounding connective tissues that stabilize the MTP joint including the plantar plate, deep transverse metatarsal ligament, flexor tendons, and lumbricals $[4,5,12]$.

When closed reduction is not possible, or if the reduction is not stable, operative reduction and stabilization is required to restore normal forefoot function, alleviate pain, and prevent complications $[4,12]$. Possible complications of delayed reduction include further swelling, ecchymosis, nerve damage and vascular damage to the skin $[8,12]$. In one case, the patient experienced avascular necrosis of the metatarsal head presumed to be due to a delay in reduction, which the authors described as analogous to hip dislocations [12]. There is also an incre- 
ased risk of impingement of surrounding neurovascular structures and potential joint damage.

Most cases in the literature use a dorsal approach for operative reduction $[2,3,5,12]$. However, this ignores the associated tear in the plantar plate. The plantar plate is critical for stability of the MTP joint, so these surgeons often need to fixate the proximal phalanx with a k-wire $[5,6]$. Our technique utilizes a plantar approach, which allows for successful reduction of the MTP joint as well as stabilization of the plantar plate. Nakano, et al. described using this approach for traumatic irreducible MTP joint, and explained that it offers the benefit of being able to look at the obstruction directly, as well as adding stability without the need for a k-wire [4]. Good results were described at two year follow-up in their case report. In plantar plate repairs, iatrogenic irritation of the digital neurovascular bundle is always a concern. Careful surgical technique, dissection, and retraction is always mandatory. However, even with such attention to detail, residual neurogenic symptoms can occur. Regardless, the plantar approach has proven to significantly reduce pain, alleviate disability, and restore function to the forefoot [14].

\section{Summary}

Our case supports the use of direct repair of a plantar plate tear because it allows for restoration of the anatomy of the durable plantar plate, adding stability to the MTP joint for tensile and compressive forces on the joint $[15,16]$.

\section{Conflicts of Interest}

There are no conflicts of interest.

All correspondence should be through Christopher W Kinter.

\section{References}

1. Bhide PP, Anantharaman C, Mohan G, Raju K (2016) A case of simultaneous traumatic dorsal dislocation of all five metatarsophalangeal joints treated successfully with closed reduction. J Foot Ankle Surg 55: 423-426.

2. Neogi DS, Bandekar SM, Sadekar V, Patnaik S, Bhat $T$, et al. (2012) Irreducible dislocation of all the lesser metatarsophalangeal joints of the foot: A Case Report. Foot Ankle Spec 5: 324-326.
3. Hibino N, Amari R, Aoki M, Yoshizumi Y, Hamada D, et al. (2010) Irreducible dislocation of the metatarsophalangeal joints of the fourth and fifth toes: A case report and anatomical study. Foot Ankle Int 31: 339-342.

4. Nakano Y, Mogami A, Kaneko K, Inoue Y (2003) Irreducible dorsal MTP joint dislocation in the second and third toes. Injury 34: 870-873.

5. Boussouga M, Boukhriss J, Jaafar A, Lazrak KH (2010) Irreducible dorsal metatarsophalangeal joint dislocation of the fifth toe: A Case Report. J Foot Ankle Surg 49: 298. e17-298.e20.

6. Stephenson KA, Beck TL, Richardson EG (1994) Plantar dislocation of the metatarsophalangeal joint: case report. Foot Ankle Int 15: 446-449.

7. McAlister JE, Hyer CF (2013) The direct plantar plate repair technique. Foot Ankle Spec 6: 446-451.

8. De Palma L, Santucci A, Marinelli M (2002) Traumatic dislocation of metatarsophalangeal joints: Report of three different cases. Foot Ankle Surg 7: 229-234.

9. Yu GV, Judge MS, Hudson JR, Seidelmann FE (2002) Predislocation syndrome. Progressive subluxation/ dislocation of the lesser metatarsophalangeal joint. J Am Podiatr Med Assoc 92: 182-199.

10. Coughlin MJ, Baumfeld DS, Nery C (2011) Second MTP joint instability: Grading of the deformity and description of surgical repair of capsular insufficiency. Phys Sports Med 39: 132-141.

11. Johnson J, Mansuripur PK, Anavian J, Born CT (2015) Closed reduction of metatarsophalangeal joint dislocations in acute and subacute presentations: A novel technique. Am J Emerg Med 33: 1333.e3-1333.e7.

12. Hey HW, Chang G, Hong CC, Kuan WS (2013) Irreducible dislocation of the fourth metatarsophalangeal joint--A case report. Am J Emerg Med 31: 265.e1-265.e3.

13. Sharma A, Dosajh R, Bedi GS, Gupta K, Jain A (2017) First and second metatarsophalangeal joint open dislocations: $A$ Case Report. Malays Orthop J 11: 71-73.

14. Prissel MA, Hyer CF, Donovan JK, Quisno AL (2017) Plantar plate repair using a direct plantar approach: An outcomes analysis. J Foot Ankle Surg 56: 434-439.

15. Gregg J, Marks P, Silberstein M, Schneider T, Kerr J (2007) Histologic anatomy of the lesser metatarsophalangeal joint plantar plate. Surg Radiol Anat 29: 141-147.

16. Johnston RB 3rd, Smith J, Daniels T (1994) The plantar plate of the lesser toes: An anatomical study in human cadavers. Foot Ankle Int 15: 276-282. 